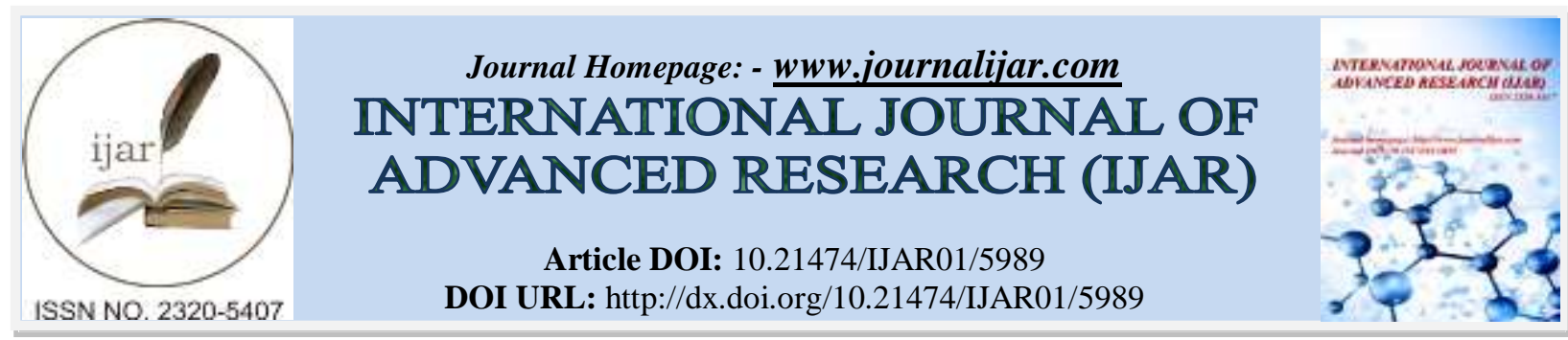

RESEARCH ARTICLE

\title{
L'ÉTHIQUE DE KANT, L'ORIENTALISME DE SAÏD ET LE SYMBOLISME DANS THE DARJEELING LIMITED.
}

Ms. ANNIE.

Research Scholar, Panjab University, Chandigarh.

Assistant Professor, Department of French, P.G.G.C.G.-11, Chandigarh.

\section{Manuscript Info}

Manuscript History

Received: 06 October 2017

Final Accepted: 08 November 2017

Published: December 2017

Key words:-

Orientalism, Symbolism, Ethics, intercultural, West.

\begin{abstract}
India is a fascinating country that has always attracted many western filmmakers. Filmmakers as diverse as Jean Renoir (Le Fleuve), Alain Corneau (Nocturne Indien), Fritz Lang (Le Tigre du Bengale), James Ivory (Shakespeare Wallah, Le Gourou) and David Lean (La Route des Indes), Claude Lelouch $(U n+U n e)$, etc. American filmmaker Wes Anderson's film The Darjeeling Limited can be read as a tribute to Satyajit Ray and makes allusions to him and his work. Co-written by Roman Coppola and Jason Schwartzman, The Darjeeling Limited includes many of Anderson's signature themes and styles, such as despair, abandonment, sibling relationships, a privileged class who rarely works, and timeless fashions and props. This film is a finelytuned critique of American materialism, emotional vacuity, and lack of spiritualism.

In this paper, I would try to look at three different aspects: firstly, what remains of India in the Western imagination after more than two millenniums of constant fantasies, fictions and stereotyping about this dream-land called India. Thus, I strive to unveil if Anderson has provided a fertile ground for engendering Orientalist cultural assumptions by producing a film using exotic locales and if he has made a significant contribution towards de-mystifying the Orient vis-àvis Edward Said's groundbreaking work Orientalism. Secondly, I endeavour to assess the value of ethics in everyday life by portraying how humanity should confront relationships with others, from inner family to complete strangers' vis-à-vis the belief system of Emmanuel Kant. Thirdly, I make an attempt to hit upon the elements of symbolism in this film.
\end{abstract}

Copy Right, IJAR, 2017,. All rights reserved.

\section{The Darjeeling Limited de Wes Anderson:-}

Ce film de Wes Anderson The Darjeeling Limited (2007) représente une contribution vers dé- mystifier l'Orient et critiquer l'Orientalisme à travers farce, auto - réflexivité et comique, résonances intertextuelles ${ }^{1}$. Ce film peut également être lu comme un hommage aux films de Satyajit Ray, qui, selon Anderson, «faisaient partie de ce qui l'a inspiré à essayer de faire des films en premier lieu $»^{2}$.

À un certain niveau, le film est un simple cliché des trois frères Whitman, Jack, Francis et Peter (Jason Schwartzman, Owen Wilson et Adrien Brody respectivement) qui se lancent dans un «voyage spirituel » à travers 
l'Inde en train ${ }^{3}$ pour se trouver et leur mère insaisissable, pour se lier l'un à l'autre, et, pour citer le caractère de Wilson, Francis, «pour dire oui à tout » (Amsden 4). À travers leurs nombreuses mésaventures et pitreries, à la fois sur et hors de l'éponyme The Darjeeling Limited, les trois hommes déplorent la mort récente de leur père, leur angoisse, et les problèmes irréconciliables de leur famille fracturée avant leur réunion. Leur brusque imprévu avec la mort alors qu'ils tentent de sauver un garçon indien conduit à une série de moments de révélation qui prévoient une possible réconciliation pour les frères, mais pas avant qu'ils ne se retrouvent à courir après un autre train, le Bengal Lancer.

\section{L'Orientalisme de Saïd et l'Inde:-}

Une lecture plus attentive du film révèle de plus grandes profondeurs et des allusions intertextuelles sous ce complot apparemment simpliste et ses éléments burlesques. D'un point de vue non-occidental, autrement dit d'un point de vue indien, je soutiens que The Darjeeling Limited tourne l'Orientalisme et ses notions inhérentes de l'exotique Est / «Autre» sur sa tête. Mes idées sur l'Orientalisme dans le contexte indien sont dues au travail révolutionnaire d'Edward Saïd en 1978, l'Orientalisme :

L'Orient était presque une invention européenne, et avait été depuis l'antiquité un lieu de romance, êtres exotiques, souvenirs et paysages envoûtants, expériences remarquables... Contrairement aux Américains, les Français et les Britanniques... ont eu longtemps la tradition de ce que j'appellerai l'Orientalisme, une façon de faire face à la Orient qui est basé sur la place spéciale de l'Orient dans l'expérience européenne occidentale. L'Orient n'est pas seulement adjacent à l'Europe ; c'est aussi le lieu de colonies les plus grandes, les plus riches et les plus anciennes, source de ses civilisations et de ses langues, concurrent, et l'une de ses images les plus profondes et les plus récurrentes de l'Autre. En outre, l'Orient a contribué à définir l'Europe (ou l'Occident) comme une image, une idée, une personnalité, et une expérience contrastées. (Saïd 1-2)

Bien que le travail de Saïd se concentre principalement sur le Moyen-Orient et le monde arabe, l'Inde a toujours été et continue à faire une partie intégrante de la représentation imaginative de l'Occident de l'altérité mystérieuse et exotique de l'Est. Saïd mentionne comment l'Orientalisme était à l'origine étroitement lié à l'impérialisme britannique et au colonialisme en Inde :

Parler d'Orientalisme, c'est donc parler principalement, mais pas exclusivement, de l'entreprise culturelle britannique et française, un projet dont les dimensions prennent des domaines aussi disparates que l'imagination elle-même, l'ensemble de l'Inde et du Levant, les Textes bibliques et les terres bibliques... L'Orientalisme découle d'une proximité particulière expérience entre la Grande-Bretagne et la France et l'Orient, qui jusqu'au début XIXe siècle avait vraiment signifié seulement l'Inde et les terres bibliques. (Saïd 4)

\section{L'Orientalisme dans le film}

L'Orientalisme comme une « style de pensée » basée sur la dichotomie entre l'Orient et l'Occident, l'Est et l'Ouest, était une création idéologique européenne si puissante et omniprésente que ses stéréotypes et ses mythes influençaient tous les registres de la connaissance, devenant « le point de départ pour des théories élaborées, des épopées, des romans, des descriptions sociales et des comptes rendus politiques concernant l'Orient, ses habitants, ses coutumes, son 'esprit', sa destinée, etc. » (Saïd 1-2). Comme le remarque Bernstein :

Les cinémas narratifs et ethnographiques occidentaux de la fin du XIXe et du XXe siècle ont hérité des traditions narratives et visuelles, ainsi que les hypothèses culturelles, sur lesquelles l'orientalisme était basé, et les cinéastes ont découvert comment l'orientalisme populaire pouvait être. (Bernstein et Studlar 3).

Dès les premiers jours du cinéma, Hollywood a fourni un terrain fertile pour engendrer des hypothèses culturelles orientalistes à travers la production des films utilisant des lieux exotiques et des femmes fatales, comme les fictions orientalistes de George Melies, le film de Douglas Fairbanks Thief of Bagdad (1924), She's a Sheik (1927) de Paramount, et Arabian Nights (1942) d'Universal pour n'en nommer que quelques-uns dans une riche histoire de colportage cinématographique de l'Oriental. Ella Shohat observe «comment les pressions sociales qui ont abouti à l'autocensure d'Hollywood ont obligé les cinéastes à exotiser et à érotiser le Tiers-Monde » en adoptant une structure de viol-fantaisie qui échappe à la parade occidentale officielle (Bernstein et Studlar 3-5). Comme le souligne 
Bernstein, « l'Orientalisme... continue d'infiltrer de nouveaux genres et films au fur et à mesure que les décennies avançaient, les films noirs comme The Maltese Falcon (1941) et Chinatown (1974) et les thrillers d'espionnage comme la série James Bond (Bernstein et Studlar 4) se retrouvaient dans le paysage exotique. Ainsi, Hollywood et le cinéma occidental ont joué un rôle majeur dans l'orientalisation de l'Orient (Saïd 6). Le film de David Lean $A$ Passage to India (1984) et les productions de Merchant Ivory en Inde telles que Heat and Dust (1983) ont été critiqués pour avoir articulé les investissements occidentaux dans l'idéologie orientaliste, pour avoir perpétué le binaire oriental-occidental et pour avoir contribué à la mystification et à la fabrication de mythes de l'Orient par l'Occident $^{4}$.

Bernstein opine que «les critiques et les théoriciens de la culture ont pris l'orientalisme comme un intrigant et un paradigme convaincant pour la représentation de la race, de l'ethnicité et du genre dans les médias et particulièrement au cinéma » (Bernstein et Studlar 4-5). Je situe une critique de ce paradigme de l'orientalisme dans la technique d'exagération et d'hyperbole d'Anderson dans la caractérisation, les situations, la cinématographie, l'éclairage, la couleur et la mise en scène, souvent source de subversion, d'ironie et d'humour.

\section{Hyperbole et hyper-réalité comme une critique}

J'affirme que l'utilisation exagérée des signifiants " occidentaux » usés par le temps de l'indianité - les images iconiques du serpent, de l'éléphant, des énormes guirlandes, des séduisantes femmes indiennes - c'est la façon dont Anderson se moquait des représentations stéréotypées de l'Occident de l'intrigue et de la mystique que symbolisent l'Inde et l'Orient. Il montre la stupidité et la futilité innées des Occidentaux qui sont assez naïs pour se lancer dans un soi-disant voyage spirituel de découverte de soi à travers «l'Orient mystique, l'Inde spirituelle » qui ne peut mener à quoi que ce soit car il est voué à l'échec et au néant dès le début. L'objectif énoncé par Francis est de « se trouver et de mieux se lier » en visitant des temples et des lieux spirituels, ce qui est au mieux un objectif destructeur et pourrait expliquer le sentiment de vide émotionnel ressenti à la fin du film.

L'excès de signifiants iconiques; la texture chaude et riche; l'émeute des couleurs éclatantes (comme les intérieurs des huttes du village et du train peints en bleu vibrant), magnifiquement capturé par la photographie de Robert Yeoman; le chaos et le rythme (ou souvent l'absence de celui-ci) signifient tous peut-être les sens accrus de quelqu'un qui visite l'Inde pour la première fois. L'attention méticuleuse d'Anderson aux détails minutieux - les intérieurs ornés d'un train comblé avec l'iconographie indienne, les motifs, et les artefacts de Rajasthan peints à la main et fabriqués par des artisans locaux; la voiture-restaurant ornée de lustres avec des peintures mogholes miniatures, et des intérieurs complexes sculptés dans le bois; les scènes chaotiques et encombrées du trafic, humain et véhiculaire, et l'étalement semi-urbain créent des accents si exagérés sur l'authentique et le « réel » qu'il entre dans le domaine de l'hyper-réalité. C'est presque trop réel pour être crédible.

\section{Localiser les sources de Subversion à travers de la Farce}

D'Aristote à Bergson, la comédie dans les arts narratifs s'est trouvée dans la situation (au sens de l'intrigue), dans le langage et dans le caractère (Cohn 11). Selon le philosophe français Henri Bergson (1859-1941), qui a analysé la dialectique de la comédie dans son essai Laughter (1900), une des conditions essentielles de la bande dessinée est que le spectateur reste insensible à la condition du personnage, car le rire est incompatible avec l'émotion (ou " l'absence de sentiment ») (Bergson). Farce crée la distance au lieu de l'identification, de sorte qu'il est possible de rire à la situation des protagonistes et de leurs idiosyncrasies, des faiblesses et des folies. Bermel fait remarquer que « Farce a deux principaux mécanismes qui libèrent le rire: les personnages qui ne sont que partiellement engagés et les situations improbables dans lesquelles ils sont rattrapés » (Bermel 22). Ces mécanismes trouvent une ample expression dans l'univers excentrique d'Anderson. Éléments d'incongruité (essayant de s'intégrer en portant des foulards à l'intérieur du gudwara [temple des sikhs] qui ne font que les rendre plus apparents que les autres fidèles sans coiffes); des situations burlesques (jetées hors du train pour cacher un serpent venimeux et échouées devant une fenêtre de réservation dans l'obscurité rurale d'un village du Rajasthan appelé Dhelana); accoutrement (les étuis en cuir de designer) ; le chahut (briser un combat au poivre) et la bouffonnerie impromptue (la charade avec la ceinture chère en cuir de Francis) contribuent tous à un détachement qui permet au public d'apprécier les situations comiques dans lesquelles se trouvent les frères Whitman.

La perception de l'incongruité devient l'une des principales sources de rire. Une séquence clé représente des enfants indiens sur des toits, des spectateurs diégétiques qui assument la position du spectateur non occidental, riant de la maladresse évidente et de la déconfiture des trois frères qui sont clairement marqués comme étrangers / autres, vêtus de leurs costumes nets qui sont incongrus dans la chaleur du Rajasthan, de lourdes guirlandes pendaient comme des 
nœuds au cou et arboraient des tikkas rouges sur leurs fronts. Observant comment « la farce se déroule dans des cadres qui maintiennent le caractère instable » (Bermel 33), Bermel explique comment un personnage burlesque est souvent placé par son auteur (ou directeur dans ce cas) :

... sur un terrain inconnu où il semble étrange et en infériorité numérique... Il est différent de tout le monde. Ses vêtements, ... son accent, ses manières peuvent le faire ressembler à intrus... Cette sorte d'incapacité, étant perdu ou ne pas appartenir, singularise le personnage quand il préfère rester discret. (Bermel 24-25)

Les frères Whitman ont des nuances des frères Marx ${ }^{5}$ à leur sujet dans leurs jeux de chevaux et de bouffonneries, dont des exemples de gestes cérémoniels bizarres, de positions absurdes yogiques et de rituels impliquant les plumes de paon et les pierres lancées sur le train qui disparait ${ }^{6}$. Comme le remarque Bermel, «La farce bafoue les limites de la raison, du bon goût, de l'équité et de ce que nous pensons généralement à la santé mentale ». (Bermel 21)

The Darjeeling Limited est une comédie de caractère et de situation qui crée des protagonistes bizarres empêtrés dans des complications amusantes, principalement de leur propre fabrication. Les physionomies contrastées, les physiques et les tempéraments des trois frères - la mélancolie frémissante de Jack et de Peter et la nature agitée et tourmentée de Francis - pris dans des situations irréelles et un «environnement tremblante » produit l'effet comique souhaité. (Bermel 33)

Outre la farce, le film s'inspire d'autres traditions comiques telles que les éléments tirés du «théâtre de l'absurde », comme en témoignent les pièces de Samuel Beckett (Cohn 11-17), une bande dessinée incarnée par le train qui se perd au milieu du désert, menant à la réalisation ironique et épiphanique par Francis: "Nous ne nous sommes pas encore localisés » :

Jack: Qu'est-ce qu'il a dit?

Peter: Il a dit que le train était perdu.

Jack: Comment un train peut-il être perdu? C'est sur les rails.

Au fil du film, la chanson mélodieuse de Peter Sarstedt, «Où allez-vous à ma belle », est imprégnée de résonance comique alors que le public reconnaît que la chanson fait partie intégrante de la routine de séduction de Jack. Le refrain idiosyncratique de Francis « Pouvons-nous accepter cela? » devient une autre source de rire qui est révélée plus tard comme un trait hérité de sa mère.

On peut soutenir que The Darjeeling Limited se rapproche de « se noyer en excentricité », une esthétique définie par Michael Hirschorn dans Atlantic Monthly comme « serrement de l'étrange contre le courant dominant ». Citant les films d'Anderson comme exemple, Hirschorn affirme que le problème avec le bizarrement est qu'il «peut rapidement passer d'un outil narratif efficace à une fin en soi » (Amsden 3). Cependant, il y a une critique formulée dans la frivolité apparente que le caprice apporte à ce film, qui est un pastiche qui emploie les pratiques parodiques d'autoréflexivité et d'intertextualité pour offrir une autre façon de regarder l'Est / l'Inde, où la lutte pour la survie oblige les enfants de risquer leur vie pour gagner à peine de quoi vivre (gagner leur vie) (symbolisé par le cireur de chaussures qui s'enfuit avec l'un des précieux mocassins de Francis).

\section{Objets en tant que caractères}

Selon Bermel, «la farce réduit la différence de conscience entre les objets et les personnes » (Bermel 27). L'utilisation fétichiste des objets dans le monde d'Anderson est, bien évidemment, à but comique, et conforme à la tradition de la farce où les « objets sont des acteurs potentiels », jouant un rôle thématique continu; récurrents dans et hors de l'action, tout comme les personnages font (Bermel 28-29). Certains des objets qui réapparaissent thématiquement dans l'univers excentrique d'Anderson sont les onze valises en cuir conçues par Marc Jacobs pour Louis Vuitton; les lunettes du père décédé, les clés de voiture, les rasoirs, et plus tard, à travers d'un flashback, sa Porsche ; le parfum, Voltaire $\mathrm{n}^{\circ} 6$; la ceinture en cuir de $6000 \$$ de Francis ; et les mocassins de $3000 \$$ (tous des signifiants évidents de la décadence et du consumérisme), les comprimés Hyno-aid ; Narco Cough, la bouteille d'analgésique de Francis ; la boîte contenant le serpent, peinte avec l'insigne du crâne et des os, qui devient l'appendice de Peter ; l'imprimante et la machine à laminer ; et le spray au poivre utilisé pour briser une bagarre fraternelle. 


\section{Dé-Orientaliser la Mystique de la Femme Orientale}

Le film explose aussi le mythe de l'insaisissable, séduisant et pourtant paradoxalement chaste femme indienne / orientale. Comme Saïd l'a résumée dans son travail en 1985, « ... l'Orient était décrit couramment comme féminine, ses richesses aussi fertiles, ses symboles principaux les femmes sensuelles » (Berstein et Studlar 3). Les marqueurs manifestes de Rita de l'indianité - ses traits terreux et sensuels et son teint accentués par ses énormes yeux rayés de khôl - confondent des représentations orientalistes de femmes pendant qu'elle fait l'amour avec un étranger (Jack) dans une toilette, fume, parle couramment l'anglais, et a un petit ami (l'insondable, attrapeur de serpent, chef steward du train joué par Waris Ahluwalia) qu'elle projette rompre avec. Elle parle franchement, elle est impatiente quand Jack veut lui parler, et montre plus de sens que lui en couvrant leur rendez-vous galant dans les toilettes. Ceci contraste fortement avec l'analyse de Saïd de la rencontre de Flaubert avec une courtisane égyptienne qui, selon lui :

«... a produit un modèle largement influent de la femme orientale ; elle n'a jamais parlé d'elle, elle n'a jamais représenté ses émotions, sa présence ou son histoire. Il a parlé et l'a représentée. Il était étranger, relativement riche, mâle, et ceux-ci étaient des faits historiques de domination qui lui ont permis non seulement de posséder Kuchuk Hanem physiquement, mais pour parler pour elle et dire à ses lecteurs de quelle façon elle était "typiquement Oriental". » (Saïd 6)

Rita, joué par la pulpeuse Amara Khan, détruit les attentes romantiques traditionnellement associées à la femme orientale par la banalité de sa rencontre sexuelle. Ainsi, son apparence indienne « authentique » traditionnelle est incompatible avec son comportement « occidental», et ce contraste est une autre source d'ironie comique.

\section{Huston comme Nonne: l'intertextualité comique et orientaliste}

Le rire naît de l'association comique - le casting d'Angelica Huston comme la nonne invraisemblable rappelle la comédie noire de The Addams Family (1991) et The Addams Family Values (1993), des films qui sont, de manière assez significative, sur des membres dysfonctionnels et excentriques de la famille comme les relations filiales et les jalousies entre frères et sœurs et la rivalité illustrée dans The Darjeeling Limited. Huston porte un bagage intertextuel pour le spectateur averti qui discerne tout de suite que son zèle évangélique ne peut être pris au sérieux.

La dimension orientaliste pour les missionnaires chrétiens en Inde qui se sont trouvés portant le « fardeau de l'homme blanc » pour éduquer les indigènes est importante à mon argument. Les missionnaires chrétiens ont longtemps été reconnus pour leur rôle dans l'exotisation de l'Orient et dans le renforcement de "la relation entre l'Occident et l'Orient [qui] est une relation de pouvoir, de domination, de divers degrés d'hégémonie complexe » (Saïd 5). Ella Shohat fait référence à la « mission civilisatrice » des nonnes britanniques en Inde, telle qu'elle est décrite dans le film orientaliste de Powell et de Pressburger, The Black Narcissus. (Bernstein et Studlar 40). En lançant Huston comme nonne, Anderson se moque de ce discours orientaliste et sape les idées de l'Occident :

... création d'une série d'images stéréotypées, selon lesquelles l'Europe (l'Occident, le 'soi') est considéré comme étant essentiellement rationnel, développé, humain, supérieur, authentique, actif, créatif et masculin, tandis que l'orient (l'Orient, 1 '« autre ») (une sorte de substitut, version underground de l'Occident ou le 'soi') est considéré comme étant irrationnel, aberrant, arriéré ou bien rétrograde, brut, despotique, inférieur, inauthentique, passif, féminin et sexuellement corrompu. (Macfie 4)

\section{Cherchant désespérément le salut}

Un tournant dans le récit sinueux se produit lorsque le trio chance sur trois garçons dont le radeau chavire dans une rivière enflée. Malgré la tentative des frères de les sauver, Mukesh, l'un des garçons, se noie. Les frères portent le corps au village des garçons, où ils sont invités à assister aux funérailles. Soudain, les chamailleries et l'angoisse des frères sont remplacées par un sentiment de tristesse et une conscience de soi naissante qui colorent le reste de leur voyage, évoquant les souvenirs des funérailles de leur père un an auparavant. La mort est acceptée avec une résignation tranquille et la dignité par les villageois, peu importe à quel point il est prématuré, comme représenté par la crémation de Mukesh, que Peter a tenté de sauver de la rivière turbulente. Cette acceptation et cette démission contrastent fortement avec les tentatives ratées de Peter d'accepter la mort de son propre père. L'utilisation des lunettes de soleil et du rasoir de son père par Peter symbolise un attachement matériel excessif et le besoin de se débarrasser de son excès de bagage, à la fois littéral et métaphorique, dans sa quête spirituelle. Comme le dit Francis, ils ont « encore beaucoup de guérison à faire », à la fois physique et émotionnel. 
Le paysage indien exerce une fonction chorale, une présence primordiale et intemporelle contre laquelle les trois frères jouent leurs petites vexations. Les frères vivent le cycle complet de la mort et de la naissance, symbolisé par un nouveau-né placé sur les genoux d'un Peter réticent. Comédie surgit non seulement de la physionomie malheureuse de Peter et de la juxtaposition visuelle du frêle bébé contre son physique viril et masculin, mais aussi de la connaissance qu'il a fuit ses devoirs parentaux imminents : il va bientôt devenir père, et pourtant, il a déserté sa femme enceinte, Alice. D'où l'ironie comique émane d'une combinaison d'hilarité et de désespoir, alors que le passé de Peter le rattrape dans les circonstances les plus inhabituelles et les plus inattendues - dans un village indien rural.

Passons à l'éthique de Kant dans The Darjeeling Limited.

\section{L'Éthique de Kant dans The Darjeeling Limited}

Depuis le début du XXe siècle, le cinéma a été intégré interculturellement en tant qu'angle sociale à travers laquelle les normes et les valeurs sociétales sont représentées. Le cinéma mieux connu comme "Le septième art », est profondément enraciné dans l'interprétation artistique. La contribution morale est sans doute l'outil le plus puissant qu'un metteur en scène a pour établir un ton spécifique et le relier au cœur de son auditoire. Tel est le cas du cinéaste américain Wes Anderson et de son film The Darjeeling Limited où il expose la valeur de l'éthique dans la vie quotidienne en montrant comment l'humanité doit affronter les relations avec les autres, de la famille intérieure aux parfaits étrangers. Comme déjà dit, dans ce film de Wes Anderson, trois frères américains partent en voyage à travers l'Inde avec un plan pour redécouvrir et revigorer leurs relations les uns avec les autres et avec leurs spiritualités individuelles. Cependant, leur voyage se déroule rapidement, et leur quête réalisée méticuleusement devient une aventure ad hoc remplie de moments d'ébriété, de colère, de sexualité insolite, la mort malheureuse et, finalement, l'épanouissement spirituel. Les frères rencontrent des dilemmes éthiques remarquables en Inde, qui, à la fin, s'avèrent la clé de l'appréciation du film. D'une manière subtile et raffinée, Anderson utilise The Darjeeling Limited comme une vitrine générale pour présenter la valeur de l'éthique dans la vie de tous les jours. Plus précisément, l'éthique d'Immanuel Kant peut être comprise en relation à la renaissance « spirituelle » ou, peut-être mieux comprise, " éthique » des frères. Plusieurs parallèles à la philosophie kantienne apparaissent dans The Darjeeling Limited.

Une compréhension de base du système de croyance d'Immanuel Kant est nécessaire pour reconnaître sa présence dans le film. Dans Grounding for the Metaphysics of Morals, Immanuel Kant fonde un système moral basé sur l'impératif catégorique. Il déclare «Il n'y a aucune possibilité de penser de quelque chose dans le monde, ou même hors de celui-ci, qui peut être considéré comme bon sans qualification, sauf bonne volonté ... c'est bon en soi » (Kant 7). Kant exprime la question du devoir de soi-même et les autres en utilisant des obligations parfaites (universelles) et imparfaites (conditionnelles). Sa philosophie du devoir est la suivante : le devoir parfait d'une personne envers soi-même est de maintenir la vie, les autres doivent être véridiques; le devoir imparfait d'une personne envers soimême est de cultiver des capacités et de les utiliser sagement, et aux autres est d'aider les autres à atteindre la même chose (Kant10). Enfin, Kant commente comment il faut agir en présence des autres : «Il n'y a donc qu'un seul impératif catégorique et c'est ceci : Agissez selon cette maxime selon laquelle vous pouvez en même temps que cela devienne droit universel » (Kant 30). Ainsi, un individu auto réglementé devrait agir comme ses actions deviendront universellement acceptables pour tous les êtres humains.

Les principes de la philosophie de Kant peuvent être vus dans The Darjeerling Limited, en particulier dans la scène tragique à la rivière. Les frères approchent un groupe de trois jeunes hommes essayant de traverser une rivière sur un radeau. Un frère, Francis remarque brusquement, « Regardez ces connards ». Dans une fraction de seconde, un coup du sort s'est passé ! Le radeau saccade violemment et jette les enfants dans l'eau dangereuse et rocheuse.

Un autre des frères, Peter crie, «Allez! » Et ils courent immédiatement à la rivière et ils plongent. Chaque frère tente de sauver un enfant, et seul Peter est infructueux ; son enfant tombe de sa prise et meurt sur les rochers. Anderson présente clairement le dilemme éthique dans cette scène : les frères ont la possibilité de refuser d'aider les garçons à l'assurance de leur propre sécurité, ou risquer leur propre vie pour le bien-être des autres. Les frères démontrent des actions conformes à l'éthique de Kant : ils risquent leur propre vie pour aider les garçons. Ceci, peut-être, pourrait même être caractérisé comme un acte d'instinct humain ; le téléspectateur est témoin de la directive de Peter « Allez » sans considérable temps pour la réflexion. Néanmoins, les frères universalisent une maxime, que, comme des individus auto réglementées, nous avons le devoir d'aider les autres en péril, indépendamment de leur race, de leur statut social ou de tout autre facteur historiquement déterminant. De même, ils démontrent une bonne volonté et un devoir imparfait de d'autres, que Kant trouverait admirables (Kant 10). 
Voyant bercer l'enfant dans ses bras, Peter s'adresse à ses frères paniqués, « Il est mort. Il est mort ; les pierres l'ont tué... je n'ai pas sauvé le mien. » Plus tard, il demande à l'interprète « Pouvez-vous m'expliquer que je l'ai presque eu ? Je l'ai perdu quand nous sommes sortis des rochers ; il était trop glissant. Je l'ai eu tout le reste du temps... Je veux qu'ils le sachent. » En effet, l'éthique affichée par les frères n’est pas liée par leurs relations avec les parties impliquées, mais de pure bonne volonté et souci de l'humanité. Il est visible que Peter a des remords et des sentiments d'inadéquation pour son échec à « sauver le sien ». Sa culpabilité montre la lutte éthique et morale qu'il a été présenté avec, et comment cela a affecté sa conscience.

Anderson contribue également à ce thème artistiquement dans le contexte du film. Pendant la scène de sauvetage, les angles de la caméra sont tels que les visages individuels des enfants ne sont que très rarement vu ; Anderson a choisi de tourner la scène d'une manière que les enfants réels ne sont visibles par derrière et seulement brièvement en avant. Quand leurs visages sont visibles, cependant, c'est comme ça que le spectateur ne peut pas dire si elles sont sauvées par Peter, Jack ou Francis. Ainsi, on ne peut pas déduire, loin après la scène, quel enfant était exactement le malheureux. L'anonymat relatif des enfants témoigne de leur dignité moralité et de leur égalité individuelle. Les enfants n'ont pas besoin d'être distingués les uns des autres - peu importe qui est sauvé et qui ne l'est pas, car la dignité humaine de chaque enfant est égale, et ils méritent tous la sécurité de la crise.

Kant argumente le point de la dignité morale et de la dignité humaine tout au long de son fondement pour la métaphysique de la morale. Il déclare : «L'impératif pratique sera donc le suivant : Agissez de telle manière que vous traitez l'humanité, que ce soit dans votre propre personne ou dans la personne d'un autre, toujours en même temps qu'une fin et jamais simplement comme un moyen » (Kant 36). Les enfants sont, en eux-mêmes, des fins morales. Pour cette raison, les enfants ne gagnent pas vraiment l'identité individuelle pendant cette scène ; leurs identités sont sans importance et, sans doute, non pertinentes. Pendant la scène, la panique, l'identité humaine est subordonnée à l'importance de l'accomplissement de l'instinct humain de base, le souci de sauver la vie d'autrui.

Ensuite, berçant l'enfant décédé dans ses bras, Pierre demande à ses frères: «Quel est son nom? » Peter, dans sa culpabilité et son échec, souhaite étendre l'identité à l'enfant, et payer le respect aux morts. L'identité est maintenant nécessaire pour traiter l'enfant avec la dignité humaine.

Par contraste, Anderson se concentre beaucoup sur les visages et les expressions des frères à ce moment dans le film. Grâce à un zoom de caméra lourd et de la musique chaotique, il montre leur peur, la panique, et le chagrin pendant la situation. Anderson choisit d'afficher les enfants comme des égaux éthiques êtres humains, mais met davantage l'accent sur les acteurs éthiques réels et leurs sentiments dans la scène. Kant décrit cette situation comme « la douleur de l'humiliation de nos inclinations » (Kant 24). Les frères doivent agir contre leurs inclinations pour se protéger, et risquer leur vie - c'est leur devoir vers les enfants et leur action éthique. Cette douleur et cette souffrance sont clairement affichées dans leurs expressions et leurs actions à la caméra. Par coïncidence, la musique chaotique s'arrête également lorsque l'enfant semble mort ; l'obligation des frères d'agir contre leurs inclinations naturelles se termine à cette jonction, et un silence un peu paisible est prédominant. La scène, prise comme une somme, démontre la résignation chaotique de soi qu'un humain subit dans le sacrifice, et fournit la philosophie de Kant avec un visage et un acte.

Arrivons au Symbolisme dans The Darjeeling Limited.

\section{Le Symbolisme dans The Darjeeling Limited}

Pour saisir la vraie beauté du Darjeeling, on doit comprendre les symboles présentés tout au long du film. Il y a beaucoup de symbolisme dans ce film, mais les trois éléments principaux sont : les trois plumes, la ceinture et toutes les valises portées par les frères. Chaque objet porte un message symbolique, qu'on peut voir sur l'interprétation étroite du film.

Chaque frère est présenté avec une plume. Ces plumes peuvent représenter des différentes choses ; mais l'un d'entre eux est la façon dont vous ressentez, la façon dont la vie se passe. Quand ils accomplissent le rituel, nous pouvons clairement voir que chaque frère ressent différemment la façon dont va leur vie. Francis Whitman (Owen Wilson) exécute le rituel correctement, symbolisant qu'il est excité par son avenir, et gardera son passé dans le dos de son esprit (en enterrant la plume sous un rocher). Francis estime que son voyage spirituel le changera pour le mieux. Peter Whitman (Adrien Brody) ne ressent pas la même chose pour sa vie. Non seulement il ne brûle pas la plume, 
mais il la garde. Cela symbolise le sentiment d'être incomplet. Avec un bébé attendu dans les 6 prochaines semaines, il se sent mal à l'aise et confus, ne sachant pas où va sa vie. Peter a peur de laisser son passé et d'avancer. Il craint un changement majeur dans sa vie, et a peur de sortir de sa zone de confort, alors il garde la plume. Enfin, Jack Whitman (Jason Schwartzman) laisse la plume se faire emporter par le vent. Cela représente le malheur et la colère. Jack veut oublier son passé et commencer une nouvelle vie. Il ne veut pas s'impliquer dans les conversations, et écrit des histoires courtes pour l'aider à aller dans la bonne direction (chercher le bonheur). Les histoires ont été décrites par Peter pour avoir une nature en colère. Mais le vent ne peut prendre une plume que jusqu'à présent, et Jack devra éventuellement affronter ses sentiments pour continuer.

Le deuxième symbole : la ceinture. Francis porte une ceinture précieuse, qui représente l'amour. La ceinture porte de vieux souvenirs affectueux de son passé; et parfois, il donnait la ceinture à Peter, prêt à abandonner ses vieux souvenirs pour en créer de nouveaux. Cependant, lorsque Francis se sent malheureux / déprimé / en colère, il cherche encore le vieil amour en enlevant la ceinture à Peter. Cela arrive plusieurs fois tout au long du film, symbolisant que Peter n'est pas encore prêt à abandonner un objet matériel d'amour. Chaque fois que Francis donne sa ceinture à Pierre en permanence, c'est un symbole qu'il est heureux, et a créé de nouveaux souvenirs affectueux sur son voyage. Il passe aussi son amour à Peter, qui en a désespérément besoin. Comme on dirait, « Laissez la ceinture travailler pour vous comme elle l'a fait pour moi ».

Un élément supplémentaire du symbolisme dans The Darjeeling Limited, ce sont les valises des frères. Chaque valise représente les vies passées des frères Whitman. Que ce soit des souvenirs heureux, tristes ou neutres. Chaque fois qu'ils voyagent, ils gardent toujours leurs valises à proximité. Cependant, comme ils vivent un changement spirituel vers la fin du film, ils n'ont plus besoin de leur passé. En courant pour attraper un train, ils jettent tous leurs valises par terre, les laissant derrière eux. C'est le tournant The Darjeeling Limited, symbolisant que tous les trois frères ont vécu un changement spirituel et sont prêts à faire une nouvelle vie pour eux-mêmes. Peu de temps après, ils accomplissent correctement ensemble le rituel de la plume, le symbole du vrai changement.

\section{Conclusion:-}

Pour conclure, The Darjeeling Limited est un film basé sur le symbolisme, avec un message derrière chaque ligne, personnage et événement. Mais ce film est censé d'être une œuvre d'art, pas un film divertissant. C'est un symbole de la vie et des choses que nous faisons en tant que peuple pour changer les nôtres. C'est un bon changement par rapport au film à succès typique.

En outre, en ce qui concerne Orientalisme, Saïd observe comment les médias sont complices de la perpétuation du mythe de l'Orient : «Un aspect du monde électronique, postmoderne, est le renforcement des stéréotypes qui soustendent l'Orient. La télévision, les films et toutes les ressources des médias ont forcé l'information dans des moules de plus en plus standardisés » (Saïd 26). Cependant, malgré cette prédilection des médias pour les stéréotypes culturels, Anderson réussit à se moquer du prisme orientaliste par lequel l'Inde est souvent réfractée dans les discours occidentaux ${ }^{7}$. Le film prend comme point de départ une prémisse ouvertement orientaliste de recherche de la croissance spirituelle en Inde, puis la subvertit au cours du film en ridiculisant une telle intention à travers la caractérisation comique et les situations loufoques, les actions et les réactions de ses principaux protagonistes - trois hommes blancs cherchant désespérément des raccourcis vers le salut ou le nirvana. Le traitement d'Anderson est incarné par l'articulation d'un cliché orientaliste défiguré par le personnage comique de Peter : "J'aime la façon dont ce pays sent bon. Je ne l'oublierai jamais. C'est plutôt épicé ».

En plus, The Darjeeling Limited soulève diverses questions sur l'éthique, le plus ouvertement par rapport à devoir envers les autres. En dirigeant le film, Anderson expose ces dilemmes et fait leur impact apparent pour le spectateur. Son commentaire sur l'éthique dans la scène à la rivière est significatif et poignant, mais subtil. Ces situations engendrent des questions d'approfondissement dans l'esprit de tout spectateur, et éclaircir les questions quelque peu insaisissables de la moralité.

\section{Commentaires:-}

1. Outre l'intertextualité comique dans le film, il est rempli d'allusions, visuelles, sonores et situationnelles, aux films du célèbre réalisateur indien Satyajit Ray, en particulier à Sonar Kella (1974) ou la "Golden Fortress », qui est basée sur un roman policier écrit par Ray en 1971 et situé dans les régions du désert du Rajasthan de Jodhpur et de Jaisalmer. Le visage bandé de Francis rappelle le blessé Docteur Hajra, qui porte des pansements 
identiques à Sonar Kella, et le voyage en train à travers le Rajasthan (Robinson 234) rappelle les aventures du détective Feluda, son neveu adolescent Topse et le personnage comique, Jatayu rencontré dans un train en route vers la «Golden Fortress» éponyme à Jaisalmer et dans les déserts du Rajasthan. La bande-son de The Darjeeling Limited est une compilation de la musique de plusieurs films de Ray tels que Teen Kanya (1961), Charulata (1964), Jalsaghar (1958) et Joi Baba Felunath (1978) ; et Merchant Ivory productions, The Householder (1963), Shakespeare Wallah (1965) et Bombay Talkie (1970).

2. Mentionné dans le livret accompagnant le CD audio de la bande son originale pour The Darjeeling Limited. Par une coïncidence étrange, Anderson a été inspiré de tourner en Inde après avoir visionné le film de Jean Renoir, The River, en 1951, sur l'ordre de Martin Scorsese, qui est le même film qui a inspiré un jeune Ray à se développer son œuvre néo-réaliste tout en observant Renoir pendant son tournage (Robinson 69). Ray a souvent mentionné l'influence considérable de Renoir sur son art dans ses écrits et ses biographies. L'utilisation de la riche palette de couleurs indiennes par Anderson est à la fois un hommage et une appropriation du Technicolor de Renoir. Voir la biographie de Marie Seton, Portrait of a Director: Satyajit Ray (1971) et le livre de Ray, Our films, Their films (1976) pour plus de détails.

3. Wes Anderson a probablement déjà été ou entendu parler du «Palace on Wheels », un train de luxe similaire à l'Orient Express, qui relie Delhi au Rajasthan et que seuls les touristes étrangers aisés peuvent se permettre. La mise en scène ressemble beaucoup aux intérieurs ostentatoires du « Palace on Wheels ».

4. Voir Parama Roy, «Reading Communities and Culinary Communities: The Gastropoetics of the South Asian Diaspora », Positions: East Asia Cultures Critique 10.2 (2002).

5. Selon Albert Bermel, «Les frères Marx ne sont pas à leur place; ils semblent même regretter d'être coincés les uns avec les autres », un peu comme les frères Whitman (Bermel 25).

6. Cela fait une référence directe à une situation dans Sonar Kella lorsque le détective, son side-kick et le personnage de bande dessinée se retrouvent bloqués, décrits par le biographe de Ray : « scènes autour d'une petite gare au milieu de nulle part, comme le trio s'installe à une longue attente pour le train qui est dû dans les petites heures » (Robinson 236) qui transpose à Dhelana d'Anderson.

7. Pour les constructions orientalistes de l'Inde, voir Ronald Inden, 'Orientalist Constructions of India', Modern Asian Studies, 20, 3, 1986; Aijaz Ahmad, 'Between Orientalism and Historicism', Studies in History, 7, 1, n.s., 1991; Carol A. Breckenridge and Peter van der Veer, Orientalism and the Postcolonial Predicament (Philadelphia: University of Pennsylvania Press) 1993; and, Richard King, Orientalism and Religion (London: Routledge) 1999.

\section{Filmographie:-}

The Darjeerling Limited. (2007). Dir. Wes Anderson. With Adrien Brody, Jason Schwartzman and Owen Wilson. Fox Searchlight.

\section{Bibliographie:-}

1. Amsden, D. (2007). The Life Obsessive With Wes Anderson. New York Magazine, 24. http://nymag.com/movies/filmfestivals/newyork/2007/38024/index2.html

2. Bergson, H. (2007). The Project Gutenberg E-text of Laughter: an essay on the meaning of the comic. http://www.gutenberg.org/dirs/etext03/laemc10.txt

3. Bermel, A. (1982). Farce: A History from Aristophanes to Woody Allen. New York: Simon and Schuster.

4. Bernstein, M., \& Studlar, G. (Eds.). (1997). Visions of the East: Orientalism in film. Rutgers University Press.

5. Cohn, R. (1959). The Comedy of Samuel Beckett:" Something old, Something New...". Yale French Studies, (23), 11-17.

6. Edelstein, D. (2007). “Clickety Clack.” New York Magazine. http://nymag.com/movies/reviews/38019/

7. Kant, I. (1993). Grounding for the metaphysics of morals: With on a supposed right to lie because of philanthropic concerns. Hackett Publishing.

8. King, R. (1999). Orientalism and religion: postcolonial theory, India and 'the mystic East'. London: Routledge.

9. Macfie, A. L. (ed.). (2000). Orientalism: A Reader. Edinburgh: Edinburgh University Press.

10. Robinson, A. (2001). Satyajit Ray: the inner eye: the biography of a master film-maker. IB Tauris.

11. Said, E. W. (2003). Orientalism. London: Penguin.

12. Shohat, E. (1991). Gender and culture of empire: Toward a feminist ethnography of the cinema. Quarterly Review of Film \& Video, 13(1-3), 45-84. 